\title{
ABERRANT OPTIC NERVE FIBRES FOUND BETWEEN RETINA AND HEXAGONAL CELLS*
}

BY

\section{Professor ARnold Loewenstein (Prague) $\dagger$}

NERVE fibre bundles of different size aberrant from the optic nerve escape according to Ida Mann (1937) from the confines of the optic stalk, and become free in the orbit. They originate at an early stage (about $17 \mathrm{~mm}$. length) when the nerve fibres find their way from the ganglion cells into the optic stalk. Anatomically they are a division of the main stem of the optic nerve in its orbital or intracranial course. In the case of Sneed (1914) which I have observed with this author the right optic nerve was split in front of the chiasma into a smaller temporal and a thicker nasal thread. Each had its own sheath. In the case of Scheerer (1923) the optic nerve was considerably broadened behind the cribriform plate. Histologically this author could prove that the nerve fibres had left the optic nerve through gaps in the pia in the shape of bundles of the optic nerve are described by $\mathrm{E}$. Fuchs, Ganser, and others. Von Szily (1924) has modelled such aberrant fibres in his reconstruction of the eyes of rabbit embryos of a coloboma strain. The aberrant bundles appear at the outside of the eye, but are to be found among the cells of the pigmented epithelium as well.

Nothing is known so far about aberrant nerve fibres of the optic nerve closely behind the retina. As I have observed two cases of entirely different aetiology and there are interesting biological questions linked with this phenomenon, the anatomical findings should be mentioned.

\section{First Case}

L., Max, aged 46 years, had always normal vision. Was a soldier and had to shoot aiming with his right eye. Lues admitted 20 years ago, treated locally only. His vision of the right eye was failing two years ago, treatment : 10 injections of $\mathrm{Hg}$ and $\mathrm{Bi}$; some intravenous injections of salvarsan. Hair dark brown, irides chestnut, without warts. Anterior halves of both eyes normal. Media clear. Right pupil 4, left $2 \frac{1}{2} \mathrm{~mm}$., both slightly irregular, positive Argyll Robertson. Right fundus (Fig. 1): The nasal $2 / 3$ of the disc is occupied by a dark, nearly black growth promi'nent over the unpigmented area-nasally the black tissue transgresses the margin of the disc slightly. The prominence of the

* Received for publication, April 28, 1944.

† From the Tennent Institute of Ophthalmology, University of Glasgow (Professor W. J. B. Riddell). 


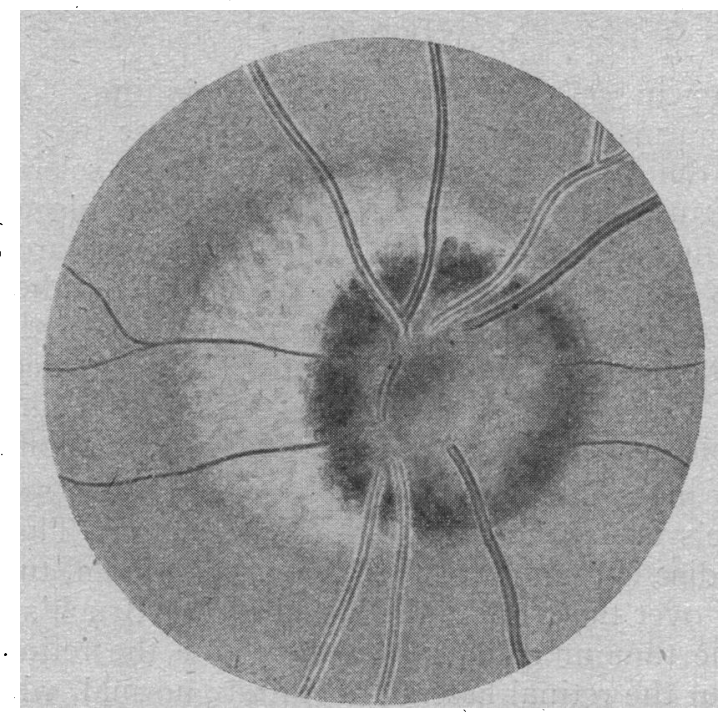

FIG. 1.

Melanotic tumour of the disc. The tumour infiltrates slightly the retina. The tissue of the unimpaired disc is out of focus.

most superficial greyish fluffy tissue is about $3 \mathrm{D}$. compared with the temporal disc tissue (which appears out of focus with the StereoGullstrand-ophthalmoscope). The vessels-arteries and veinshave a thin whitish sheath. Fundus towards the periphery tesselated.

Left eye is normal except for a typical Argyll Robertson pupil.

Vision : R. eye, fingers at $3 \mathrm{~m}$. L. eye, $6 / 5$ no + glass.

Field of vision: $R$. eye : much contracted from all sides except temporal (with $5 \mathrm{~cm}$. mark), but even $10 \mathrm{~cm}$. mark colours not recognized. L. eye : normal.

Urine : nothing abnormal. Reaction for melanin (1) Thormaelen (2) Brahn negative.

Wa. R : +++

Meinicke $\left\{\begin{array}{l}a+++ \\ b+\end{array}\right.$

Mueller +++

Schiötz $\left\{\begin{array}{l}\text { R. } 27 \mathrm{~mm} . \\ \text { L. } 19 \mathrm{~mm}\end{array}\right.$

After three weeks energetic antiluetic treatment no improvement.

Excision of the eye January 18, 1939. No further observation possible due to external conditions. 


\section{Histology}

Embedding in celloidin-horizontal sectioning. Conjunctiva and cornea normal. Angle of anterior chamber free. The tissue in front of the widely open Schlemm's canal, scleral spur and Schwalbe's ring is densely powdered with snuff pigment. Some vessels linking Schlemm's canal with scleral veins are pigmented too. Iris is amply pigmented, the pigmented epithelium cystic (there is no diabetes). Dilatator fibres are framed with slim stroma pigment cells. Sphincter fibres are powdered with pigment dust. Pigmented epithelium is thickened in the periphery of the choroid, especially in places of Blessig-Iwanoff's cysts.

The black mass fills about $3 / 4$ of the optic cross section. The central vessels are enveloped by the black mass. The nasal part of the optic disc is bulged forward like a mushroom, tumour tissue pushes itself over the hyaline thickened end of Bruch's membrane. As far as the tumour reaches into the retina the retinal structure is upset. But the retinal layers are entirely normal, where there is no tumour. tissue. The melanotic masses infiltrate the choroid somewhat deeper (about $1000 \mu$ ) (Fig. 2).

The tumour leaves temporally a strip of optic nerve tissue uncovered, the breadth of which varies between $130-450 \mu$. The black masses perforate the cribriform plate (Fig. 3). There the diameter of the cross section of the optic nerve is doubled.

At the temporal margin of the disc the retina is detached from the pigmented epithelium by a broad layer of unpigmented nucleated fibres which originate in the optic nerve as each section demonstrates (Fig. 4). They run in arcs from the retinal nerve fibre layer on one side and from the optic nerve on the other. They turn sharply at both ends of the base of the triangle inwards into the triangle and converge slightly to the acute angle at the edge. They are at the broadest spot of the triangle, the end of the pigmented epithelium, abdut 20 layers thick. The whole triangle is $700 \mu$ long. But this well stained nerve fibre tissue is continued another $650 \mu$ farther subretinally by a less well stained, less differentiated tissue. The retina over the subretinal growth consists of the anterior layers only. No outer granular nor outer nuclear layer is present within $600 \mu$ from the disc. From there on for $750 \mu$ the outer nuclear layer is represented by one row of nuclei only, which are remarkably big. This cell type may perhaps correspond to the cone nuclei. The maldevelopment of the outer retinal layer is limited exactly to the extent of the subretinal growth. A very delicate line may be the remnant of the outer limiting membrane. Retinal structure close to the malformation is completely normal including the neuro-epithelium. Pigmented epithelium-as far as 
a cross section can tell-is normal. Ciliary nerves perforating the sclera obliquely are heavily pigmented (Fig. 5).

At the depigmented slide the tumour cells are polyhedric (Fig. 6) with oval nucleus, a slightly granular plasma and visible cellular boundaries. There are fine dark fibres which can be seen distributed over the whole tumour area (Fig. 7). These fibres are much thinner than the nerve fibres outside the tumour. In some areas of the tumour the nuclei are shrunken and pressed to the cellular walls, in others the cells are swollen, the nuclei missing.

\section{Discussion}

In spite of the fact that I did not save from Prague more than 11 slides of this case and no specific nerve staining was performed, I now suppose that the subretinal tissue consists of nerve fibres. These fibres can be followed up perfectly into the optic nerve, where they turn sharply.

Aberrant nerve fibre bundles of the optic nerve have not been described so far within the tissue of the human eyeball. It is of great intereșt that the retina is maldeveloped in its posterior layers corresponding to the whole extent of the subretinal growth.

We know that the inner retinal layers are built up prior to the outer layers (I. Mann, 1928). When the percipient layers (rods and cones) appear the inner layers are complete and the ganglion cells have already sent out their fibres into the cavity of the optic stalk. If some fibres aberrate and grow under the retina they may interfere with the development of the outer retinal cells.

The question is justified whether this black mass is a malignant growth at all. The depigmented cells are of a shape which we are used to link with naevus. On the other hand, the deterioration of the vision is undoubtedly of fresh date and no other reason for the failing sight was discovered by repeated examination. The optic disc of the diseased eye was as pink as the other-therefore a tabetic atrophy could be excluded in which the pallor of the disc is mostly obvious at a time when no failure of function is yet recognizable. Retina and choroid were infiltrated by the tumour. Therefore the conclusion seems justified that a growing tumour is responsible for the failing vision. Malignant degeneration of pigmented naevi is well known in skin, iris and choroid.

The presence of pigmented naevi in the optic nerve is well understandable. The optic stalk consists of the two layers of the secondary cup into which the nerve fibres of the retinal ganglion cells grow. The outer layer of the optic stalk is therefore the cell form corresponding to the hexagonal cells. It becomes converted into unpigmented (in man) neuroglial supporting tissue (I. Mann, 
1928). We understand that cells of the outer layer of the stalk having escaped conversion into neuroglial tissue may have preserved their primitive nature and the capacity of pigment production like the other cells of the outer layer of the cup. These isolated explanted cells appear as pigmented naevus and are prone to malignant growth.

We have found a second case of nerve fibres behind the retina which we have to explain in an entirely different fashion. It was discovered before the abnormality in the first case was understood and has directed actually my attention to the problem. It was the posterior half of an eye received post-mortem, a hypertensive retinopathy which we have studied with $A$. J. Ballantyne in the course of other investigations.

There was a high grade retinal oedema with fatty exudate mostly in the outer granular layer, multiple 'groups of cytoid bodies in the retinal nerve fibre layer, endothelial and subendothelial fatty necrosis of arteries and veins of retina and choroid and a subretinal exudate. The disc was markedly oedematous and many cytoid bodies were found within its nerve fibres.

A big "druse" of $240: 140 \mu$ size stained with haematoxylin dark purplish, bulges the fibres of the optic nerve over the end of Bruch's membrane (Figs. 8 and 9). The lime content is responsible for its being broken up. There is a triangular shaped process from the optic nerve with its base in front of the cribriform plate pushing itself between retina and the irregular pigmented epithelium. This tissue between retina and pigmented epithelium is $920 \mu$ long and at its base $270 \mu$ broad and consists of nerve fibres at its base which can be followed up into the nerve fibres of the optic nerve. The greater mass of this subretinal tissue is characterized by the presence of huge typical cytoid bodies.

There is no maldevelopment of the retina present in front of the tissue, except some damage to the rods and cones, always found with subretinal exudate. The cytoid bodies in the retinal nerve fibte layer and in the-retroretinal tissue are indistinguishable. There is no reason to assume in this case a congenital anomaly as in our first case. There is no maldevelopment of the posterior retinal layers. We suppose that the swollen oedematous disc with the production of the big " druse" led to a rupture of the lining of the optic nerve at the height of Bruch's membrane. The fibres of the optic nerve grew out or were pushed out mechanically by a vis a tergo from the oedematous tissue of the optic nerve into the gap between retina and hexagonal cells. They degenerated in a ganglioform manner. 


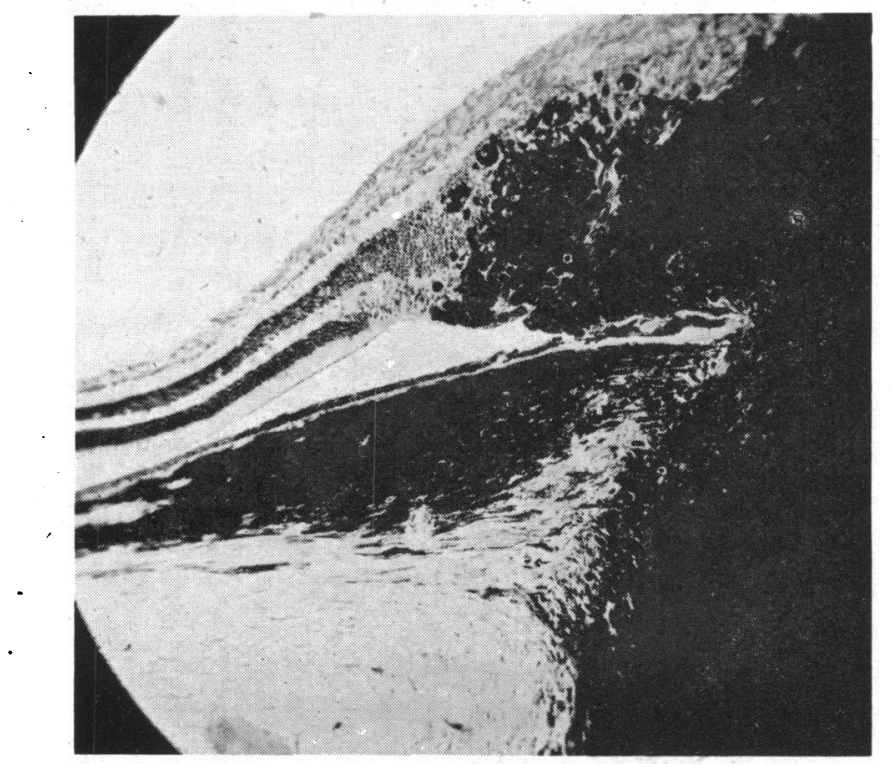

FIG. 2.

Mushroom-like growth of the pigmented tumour infiltrating retina and choroid.

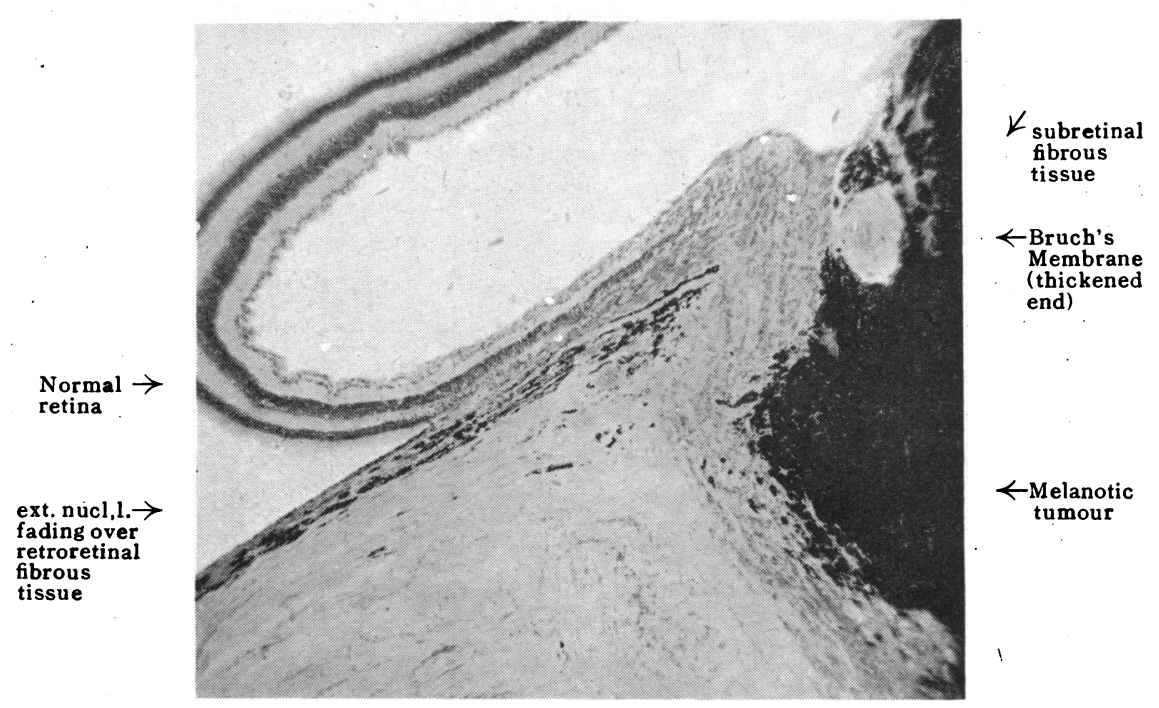

FIG. 3.

Melanoma of the disc. R.E. low power. Fig. 3 represents the other half of the disc shown in Fig. 2, the section having been mounted in obverse fashion. 


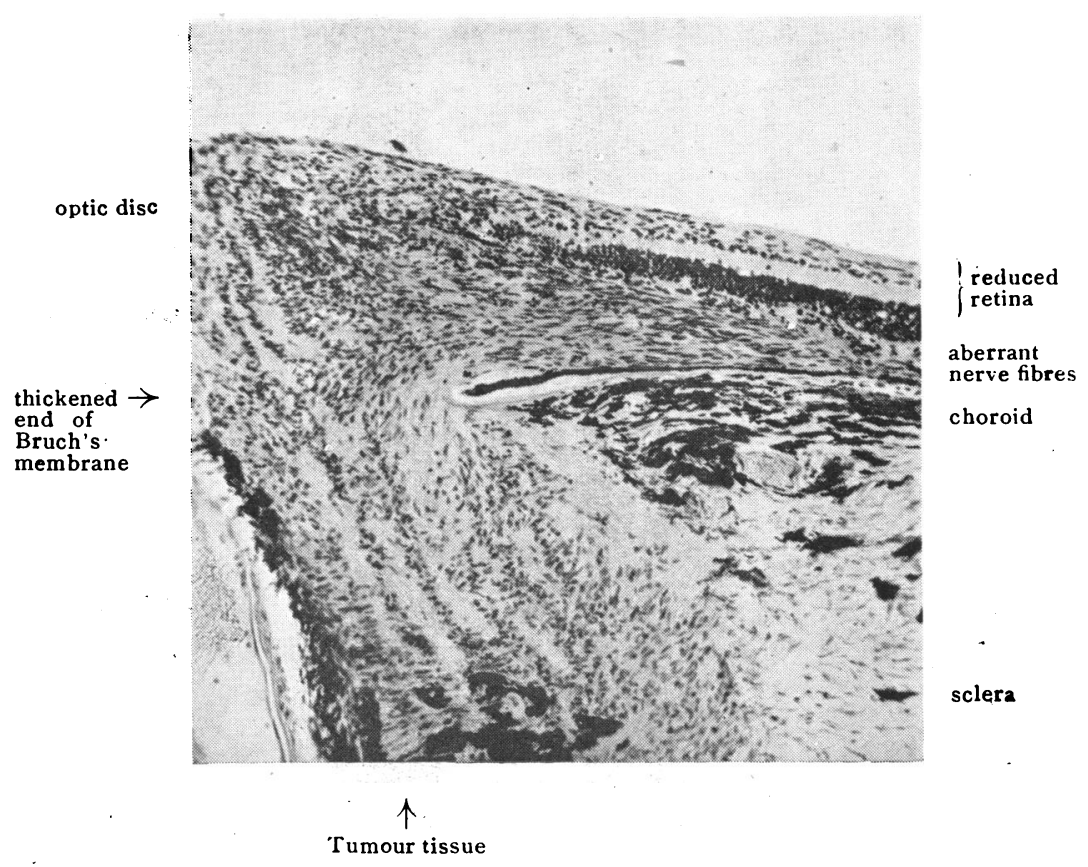

FIG. 4. High power.

Temporal part of the disc with the aberrant nerve-fibre bundles.

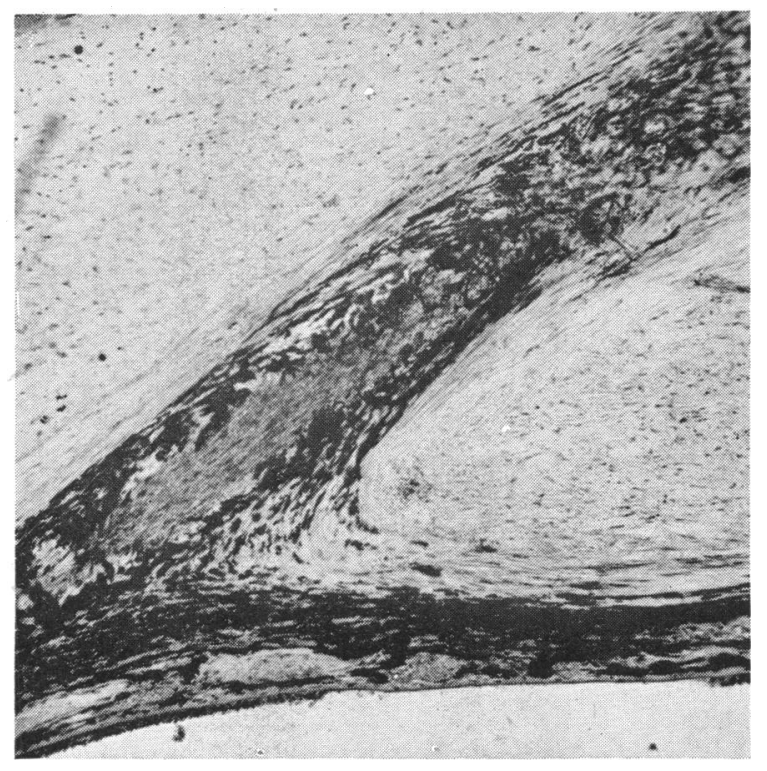

\section{posterior} ciliary nerve

FIG. 5.

A) posterior ciliary Inerve covered with stroma pigment and its insertion in locally thickened choroid. 


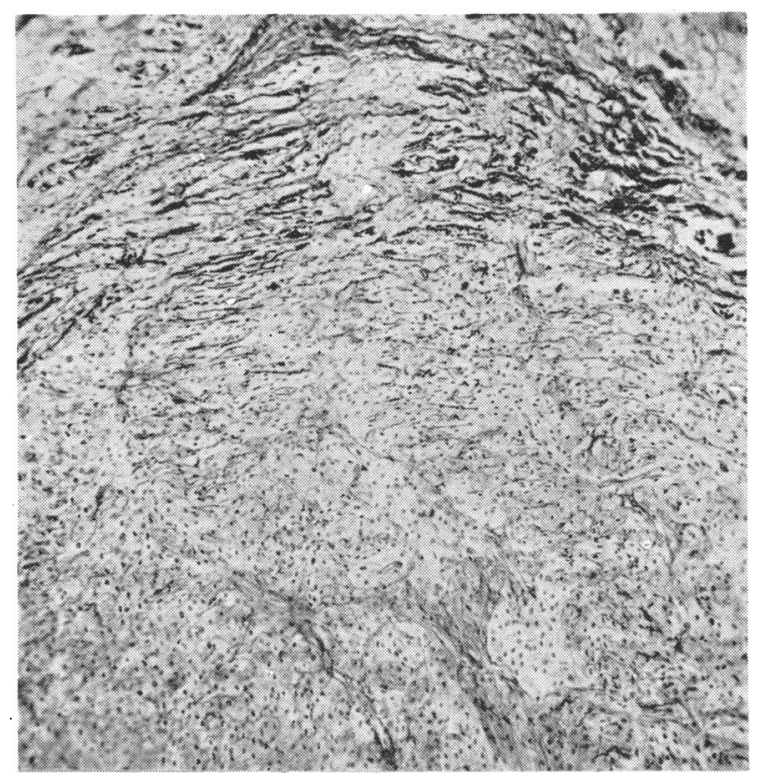

scaffolding of optic nerve

depigmented tumour

FIG. 6.

Depigmented tumour of the optic nerve towards the normal posterior part of the excised optic nerve.

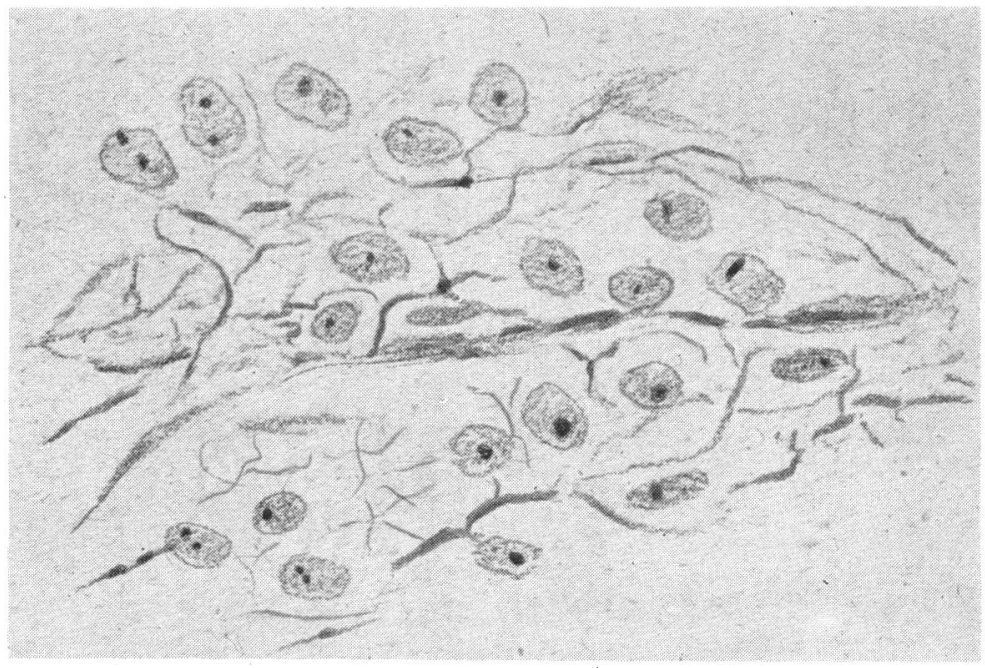

Fig. 7.

Melanotic tumour of optic nerve. Depigmented. $(675 \times)$. Note the big epithelial cells with round or polyhedric nuclei and one or two nucleoli. 


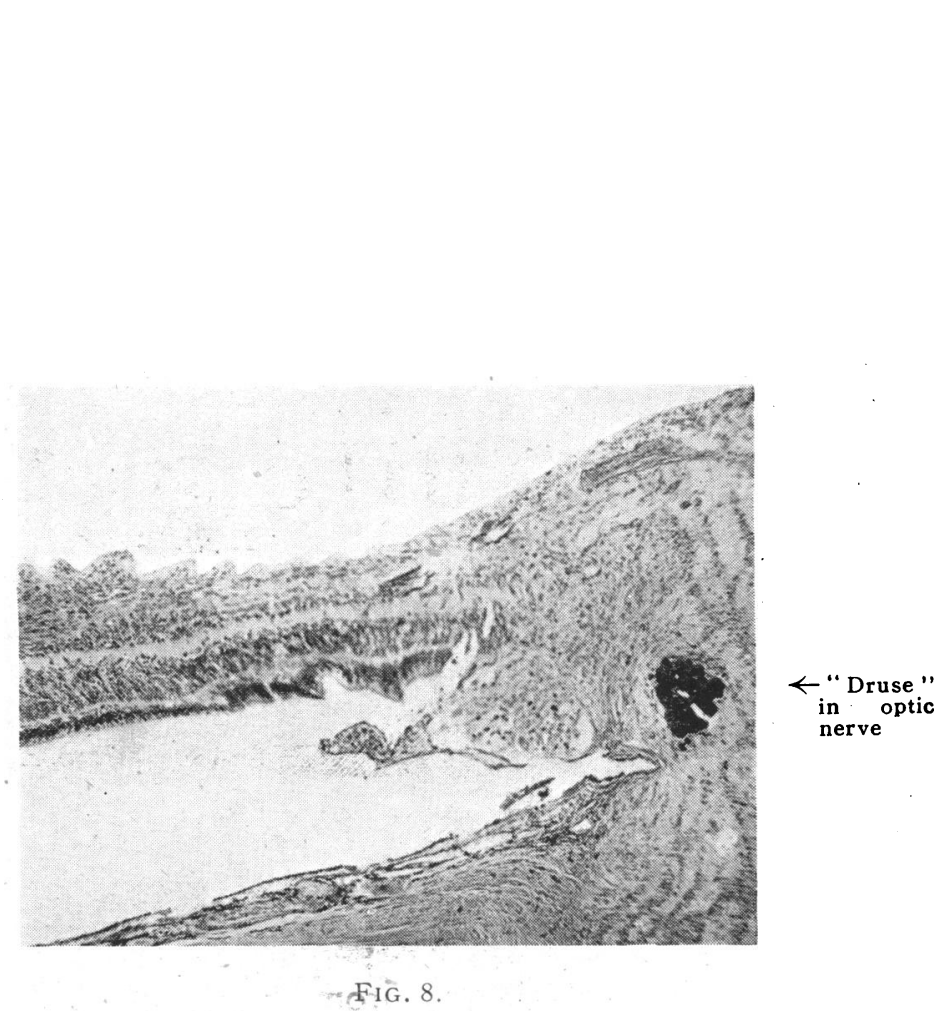

Hypertensive retinopathy. $(60 \times)$. Subretinal cytoid bodies.

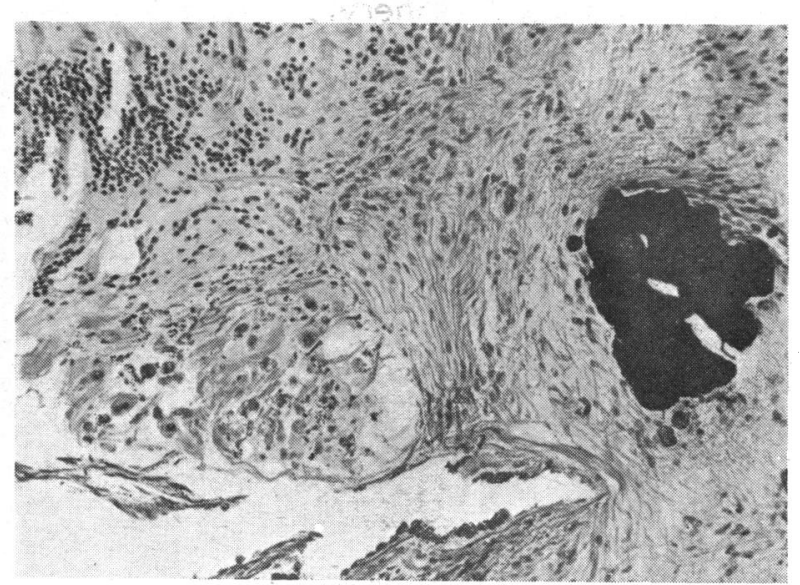

FIG. 9.

High power. $(150 \times)$. 


\title{
Summary
}

Two different kinds of aberrant nerve fibres of the optic nerve are described, they are found between retina and hexagonal cells. One type involves maldevelopment of the posterior retinal layers in front of the growth. This was a case of a pigmented tumour of the optic disc infiltrating into the neighbouring retina and choroid. It is assumed to be a malignant degeneration of a pigmented naevus of the optic disc. The second is a case of hypertensive retinopathy with sedematous swelling of the papilla and a big druse. The nerve fibre tissue found between retina and pigmented epithelium is ganglioform degenerated. The retina in front of this tissue is well developed. A mechanical pushing out of the optic nerve fibres due to the oedema and the pressure of the druse is assumed to be the cause.

\section{LITERATURE}

MANN, IDA.-Development of the human eye. Cambridge, 1928.

- Developmental abnormalities of the human eye. Cambridge, 1937.

SCHEERER. - Klin. Monatsbl. f. Augenheilk., Vol. LXXI, p. 674, 1923.

SNEED.-Arch. $f$, Augenheilk., Vol. LXXVI, p. 117, 1914.

Voy SzILY.-Zeitschr. f. Anatomie, Vol. LXXIV, p 1, 1924'(reprint).

\section{THE EFFECT OF DETERGENT ON THE PENETRATION OF SODIUM SULPHACETAMIDE (ALBUCID SOLUBLE) INTO OCULAR TISSUES*}

\author{
BY \\ M. Ginsburg and J. M.. Robson \\ (FROM THE DEPARTMENT OF PHARMACOLOGY, \\ UNIVERSITY OF EDINBURGH)
}

IT has been shown recently (Bellows and Gutman, 1943; Leopold and Scheie, 1943) that the penetration of sulphonamides applied locally to the eye can be increased by various wetting agents.

In a number of investigations (see Robson, 1944; Robson and Scott, 1944) it has been found that experimental infectitons of the cornea with various organisms can be treated successfully by the local application of a number of sulphonamides, the most satisfactory results being observed with sodium sulphacetamide. This substance penetrates readily into the cornea and does not produce any irritation. The present experiments were carried out to determine whether the penetration of sodium sulphacetamide can be

* Received for publication, November 10, 1944. 